\begin{tabular}{|c|c|}
\hline Title & Movement-related cortical magnetic fields associated with self-paced tongue protrusion in humans \\
\hline Author(s) & Maezawa, Hitoshi; Oguma, Hidetoshi; Hirai, Y oshiyuki; Hisadome, Kazunari; Shiraishi, Hideaki; Funahashi, Makoto \\
\hline Citation & $\begin{array}{l}\text { Neuroscience Research, 117, 22-27 } \\
\text { https://doi.org/10.1016/.neures.2016.11.010 }\end{array}$ \\
\hline Issue Date & $2017-04$ \\
\hline DOC URL & http:/hdl.handle.net/2115/68828 \\
\hline Rights & $\begin{array}{l}\text { () 2017. Ins manuscript verssion is made avallable under the CC-BY-IC-IVD } 4.0 \text { IIcense } \\
\text { http://reativecommons.org/icenses/by-nc-nd/4.0/ }\end{array}$ \\
\hline Rights(URL) & http:/creativecommons.org/icenses/by-nc-nd/4.0/ \\
\hline Type & article (author version) \\
\hline File Information & Manuscript.pdf \\
\hline
\end{tabular}

Instructions for use 


\title{
Movement-related cortical magnetic fields associated with self-paced tongue protrusion in humans
}

Hitoshi Maezawa ${ }^{\mathrm{a},{ }^{*}}$, Hidetoshi Oguma ${ }^{\mathrm{b}}$, Yoshiyuki Hirai ${ }^{\mathrm{a}}$, Kazunari Hisadome ${ }^{\mathrm{a}}$, Hideaki Shiraishi ${ }^{\mathrm{c}}$, Makoto Funahashi ${ }^{\mathrm{a}}$

${ }^{a}$ Department of Oral Physiology, Graduate School of Dental Medicine,

Hokkaido University, Kita-ku, Sapporo, Hokkaido 060-8586, Japan

${ }^{b}$ School of Dental Medicine, Hokkaido University, Kita-ku, Sapporo, Hokkaido

060-8586, Japan

${ }^{c}$ Department of Pediatrics, Graduate School of Medicine, Hokkaido University, Kita-ku, Sapporo 060-8638, Japan

*Corresponding author: Hitoshi Maezawa, DDS, PhD

Address: Department of Oral Physiology, Graduate School of Dental Medicine,

Hokkaido University, Kita-ku, Sapporo, Hokkaido 060-8586, Japan

TEL: 81-11-706-4229; FAX: 81-11-706-4229

E-mail: maezawa@den.hokudai.ac.jp

Short title: Readiness fields to human tongue protrusion

Number of pages: 23

Number of words in the abstract: 194

Number of figures: 4

Number of tables: 1

Number of cited references: 30

\begin{abstract}
Abbreviations:
$\mathrm{BP}$, Bereitschaftspotential; CMC, Cortico-muscular coherence; ECD, equivalent current dipole; EEG, electroencephalography; EMG, electromyogram; EOG, electrooculogram; HPI, head position indicator; M1, primary motor cortex; MEFs, motor-evoked fields; MEG, magnetoencephalography; MFs, motor fields; MRI, magnetic resonance image; post-MF, post-movement fields; RFs, readiness fields; ssMRMFs, steady-state movement-related magnetic fields; $\mathrm{S}$, primary somatosensory cortex; tSSS, temporal extension of Signal Space Separation.
\end{abstract}




\begin{abstract}
Sophisticated tongue movements are coordinated finely via cortical control. We elucidated the cortical processes associated with voluntary tongue movement. Movement-related cortical fields were investigated during self-paced repetitive tongue protrusion. Surface tongue electromyograms were recorded to determine movement onset. To identify the location of the primary somatosensory cortex (S1), tongue somatosensory evoked fields were measured. The readiness fields (RFs) over both hemispheres began prior to movement onset and culminated in the motor fields (MFs) around movement onset. These signals were followed by transient movement evoked fields (MEFs) after movement onset. The MF and MEF peak latencies and magnitudes were not different between the hemispheres. The MF current sources were located in the precentral gyrus, suggesting they were located in the primary motor cortex (M1); this was contrary to the MEF sources, which were located in S1. We conclude that the RFs and MFs mainly reflect the cortical processes for the preparation and execution of tongue movement in the bilateral M1, without hemispheric dominance. Moreover, the MEFs may represent proprioceptive feedback from the tongue to bilateral S1. Such cortical processing related to the efferent and afferent information may aid in the coordination of sophisticated tongue movements.
\end{abstract}

Keywords: magnetoencephalography; readiness fields; motor fields; hypoglossal motor nucleus; voluntary movement; MEG 


\section{Introduction}

Movement-related cortical activation associated with self-paced voluntary movement, which is known to reflect central motor control processes, has been examined using electroencephalography (EEG) and magnetoencephalography (MEG). Although examining movement-related cortical activation with EEG and MEG has shown that the most prominent reflection of finger movement is in the sensorimotor area, EEG has limited spatial resolution, as the low conductivity of the skull causes the cortical signals to spread (Vaughan et al., 1968; Deecke et al., 1969; Shibasaki et al., 1980; Cheyne and Weinberg, 1989); therefore, we used MEG in the present study.

Analysis of movement-related cortical fields (MRCFs) obtained using a whole-head MEG system during unilateral finger movement revealed that the spatiotemporal information processing of pre- and post-movement changes occurred in the primary sensorimotor cortex, with contralateral hemisphere dominance (Nagamine et al., 1996; Oishi et al., 2004). During self-paced finger movement, slow pre-movement motor fields, referred to as readiness fields (RFs), begin about 0.5-1 s prior to movement onset (Kristeva et al., 1991). They reach their maximal amplitude around movement onset, whereupon they are called motor fields (MFs). After movement onset, movement evoked fields (MEFs) appear. It has been demonstrated that MFs and MEFs originate from different cortical mechanisms: MFs mainly reflect the preparation and control of movement in the primary motor cortex (M1), while MEFs mainly reflect proprioceptive feedback from the muscles to the primary somatosensory cortex (S1) (Shibasaki and Hallett, 2006).

Although the cortex may contribute to the fine-tuning of tongue movement during speech and swallowing, little is known about the spatiotemporal cortical processes 
before and after self-paced, simple tongue movements in humans. Nakasato et al. (2001) reported the MRCFs during repetitive tongue movement and successfully analyzed the location of the tongue region of M1 in five healthy volunteers. However, in that study, the time resolution was insufficient, owing to the interindividual variability of the time delay between the trigger signal and the onset of tongue movement among the subjects; thus, the pre-movement (RFs and MFs) and post-movement components (MEFs) were not clearly discriminated. The authors recorded tongue MRCFs with a trigger that signaled when the tip of the tongue reached the optical fiber on the upper frontal part of the palate. However, using an electromyogram (EMG) trigger signal can effectively minimize the time delay between the trigger signal and tongue movement onset (Shibukawa et al., 2004). Moreover, Nakasato et al. (2001) also mentioned that the signal-to-noise ratio of the raw MRCF data was a limitation. Obtaining clear waveforms of tongue MRCFs is difficult, since the cortical magnetic fields can be contaminated by magnetic artifacts resulting from unintended muscle activity in the orofacial region. To improve the signal-to-noise ratio, the simultaneous recording of temporal EMGs and electrooculograms (EOGs) might be useful for controlling the unintended temporal and/or eye movements.

Here, we aimed to investigate the spatiotemporal cortical processes that occur in both hemispheres before and after a voluntary, self-paced simple tongue movement, using a whole-head MEG system with a tongue EMG trigger signal.

\section{Materials and methods}

\subsection{Subjects}

The MEG recording experiments were conducted in seven healthy right-handed subjects 
(5 men, 2 women; mean age, 27.1 years; range, 23-38 years). The study was performed in conformity with the Declaration of Helsinki and approved by the Ethical Committee of Dental Medicine of Hokkaido University. Written informed consent was obtained from all subjects before they were included in the study.

\subsection{MRCF recording}

Each subject was instructed to perform repetitive, brisk forward protrusion of the tongue at his/her own pace with intervals of $\sim 10 \mathrm{~s}$. These tongue movements were performed with the mouth slightly open, so the tongue did not touch the lips, teeth, or palate. During the tongue movement task, subjects were instructed not to move any other orofacial area to the greatest extent possible, including the face, jaw, and lips. Each recording session lasted from 8 to $12 \mathrm{~min}$, with several minutes of intervening breaks between sessions to refresh subjects. Each subject underwent 2-4 sessions.

Bipolar surface EMGs were recorded from the dorsum of the tongue muscles by using disposal EMG electrodes (Vitrode V, Nihon Kohden, Tokyo, Japan). Disposal tongue EMG electrodes were replaced with new ones before each session to record stable EMGs. Bipolar surface EMGs were also recorded from the bilateral temporal muscles with a pair of silver-silver chloride disc electrodes, to check for artifact contamination caused by unintended face and/or jaw movements. Conventional vertical EOGs were also recorded with a pair of silver-silver chloride disk electrodes on the right side, to detect eye-blink artifacts.

To record the magnetic fields, we used a superconducting quantum interference neuromagnetometer device with 306 channels (VectorView, Neuromag, Helsinki, Finland). The neuromagnetometer had 102 trios comprised of one magnetometer and 
two planar gradiometers that were orthogonally oriented. However, only 204 planar gradiometers were used in our analysis (Hämäläinen et al., 1993).

A three-dimensional digitizer (Isotrak, Polhemus, Colchester, VT, USA) and four head position indicator (HPI) coils, which were fixed to the forehead and the mastoid process bilaterally, were used to determine the locations of the following anatomical landmarks: nasion and bilateral pre-auricular points. The exact positions and orientations of the sensors with respect to the head were determined at the beginning of each recording session by applying currents to the HPI coils and measuring the magnetic fields they produced.

All signals were digitized at $600 \mathrm{~Hz}$ and band-pass-filtered at $0.03-200 \mathrm{~Hz}$ for MEG and at 10-200 Hz for EMG (tongue and temporal) and EOG. A subset of the MEG, EMG, and EOG signals were displayed on a screen, allowing us to monitor the subjects' task performance. The tongue movement intervals were checked using tongue EMGs. To confirm subjects’ alertness during the recording, we also monitored the subjects' behavior with a video camera, and they were interviewed about their vigilance level before and after each recording session. For determining the brain anatomy, magnetic resonance images (MRIs) of the brain were obtained for each subject with a 1.5-Tesla whole-body scanner (General Electric, Milwaukee, WI, USA).

\subsection{MRCF analysis}

The raw data were spatially filtered offline with the temporal extension of Signal Space Separation (tSSS) (Taulu and Simola, 2006; Taulu and Hari, 2009) by using the MaxFilter software (version 2.2.10, Neuromag, Helsinki, Finland). The rectified tongue EMG signals were analyzed off-line to obtain precise alignment with the EMG onset of 
tongue movement. Each EMG burst was reviewed visually, and the epochs that contained ambiguous tongue EMG bursts or temporal EMG and/or EOG activity associated with unintended temporal and/or eye movement were omitted from the analysis; then, the MEG signals and rectified EMG signals were averaged. The analysis time window was $4 \mathrm{~s}$ and began $3 \mathrm{~s}$ prior to tongue EMG onset. The time window between $3 \mathrm{~s}$ and $2.5 \mathrm{~s}$ prior to EMG onset was adopted as the baseline period. The ground averaged data of 2-4 sessions was used for further analysis. After digital low-pass filtering at $20 \mathrm{~Hz}$ for the averaged MEG signals, the signals were subjected to latency and amplitude analyses for each component. Analyses of the peak latency and amplitude were performed using the channel with the largest amplitude for each component. To determine the onset of the slow RF response, the mean of 2 standard deviations (SDs) of the baseline signal was defined as the resting activity (Nagamine et al., 1996). RF onset was defined as the intersection of the baseline with the regression line, which started when the signal exceeded the resting activity and ended at the peak (Nagamine et al., 1996).

\subsection{SEF recording and analysis}

Following electrical stimulation, we used an electrical stimulator (SEN-3401, Nihon Kohden, Tokyo, Japan) to record SEFs from the right side of the tongue. Electrical stimulation was performed using a pair of pin electrodes (400- $\mu$ m diameter), as they can deliver a low-intensity stimulus safely to a small oral area (Maezawa et al., 2014a, b, 2016a, b, 2011). The electrodes were $0.4 \mathrm{~mm}$ in diameter and the inter-electrode distance was $3 \mathrm{~mm}$. We applied tongue stimulation $1 \mathrm{~cm}$ from the tongue edge and 3-4 cm from the tongue tip (Maezawa et al., 2008). Biphasic, constant-current, square 
electric pulses ( $0.5 \mathrm{~ms}$ for 1 phase) were applied at $1 \mathrm{~Hz}$. In each subject, the stimulation intensity at each site was set to 2.5 to 3 times the sensory threshold for that stimulation site. Stimulation was applied at least 240 times. The recording passband and sampling rate were $0.1-330 \mathrm{~Hz}$ and $997 \mathrm{~Hz}$, respectively. We set the analysis window for averaging as $50 \mathrm{~ms}$ before to $500 \mathrm{~ms}$ after each trigger signal. We calculated the baseline as being from -50 to $-5 \mathrm{~ms}$ before the onset of stimulation.

\subsection{Source localization}

Isocontour maps were constructed from the data measured at the peak latency of each component (MF, MEF, and SEF). The forward-calculated (simulated) field from the first equivalent current dipole (ECD) with the orientation fixed by the projection operator was subtracted from the real (measured) field (Tesche et al., 1995; Uusitalo and Ilmoniemi, 1997) in order to analyze the data using multiple-dipole models for the MRCFs. Then, we searched for additional ECDs using the resulting complement waveform. We accepted only the ECDs that attained an 80\% goodness-of-fit. The three-dimensional location and orientation of the ECD in a spherical conductor model were determined on the three-dimensional coordinate frame detected by HPI coils. Then, the ECDs were superimposed on the subject's MR images to determine the source locations with respect to the anatomical structures.

\subsection{Statistics}

The differences between the hemispheres were statistically examined using paired t-tests for the following: (1) the latency of RF onset, (2) peak latency of the MF and MEF, and (3) peak magnitude of the MF and MEF. The ECD locations over the left 
hemisphere in each axis ( $x$-axis, $y$-axis, and $z$-axis) were analyzed for the MRCFs (MF and MEF) and SEFs using repeated measures analyses of variance with Bonferroni corrections (MF vs. MEF vs. SEF). The $x$-axis passes through the preauricular points from left to right, while the $y$-axis passes through the nasion, and the $z$-axis points upward from the plane determined by the $x$ - and $y$-axes. A p value of $<0.05$ was considered statistically significant.

\section{Results}

\subsection{Waveform configuration}

In the final off-line analysis of the data, the total sweeps number of grand-averaged data per subject was 102-168 artifact-free epochs. Fig. 1 shows an example of the whole-head, magnetic MRCF waveforms obtained from Subject 5. The pre-movement slow magnetic fields and post-movement fields were detected over the sensorimotor areas in both hemispheres. Fig. 2 depicts more detailed representative MRCF waveforms over each hemisphere, showing that the RF signals began $1.63 \mathrm{~s}$ (left hemisphere) and $0.34 \mathrm{~s}$ (right hemisphere) before movement onset, and that the RF culminated in sharp peaks (MFs) with amplitudes of $69.6 \mathrm{fT} / \mathrm{cm}$ at $37 \mathrm{~ms}$ (left hemisphere) and $-34.3 \mathrm{fT} / \mathrm{cm}$ at $41 \mathrm{~ms}$ (right hemisphere) after movement onset. Both RFs and MFs were observed over the bilateral sensorimotor areas, followed by MEFs peaking at $214 \mathrm{~ms}$ with an amplitude of $-67.3 \mathrm{fT} / \mathrm{cm}$ (left hemisphere) and at $175 \mathrm{~ms}$ with an amplitude of $59.0 \mathrm{fT} / \mathrm{cm}$ (right hemisphere) (Fig. 2).

A similar pattern of magnetic signals was observed over both hemispheres in all of the subjects. Table 1 summarizes the RF onset latency, and the peak latency and peak magnitude of the MFs and MEFs over each hemisphere in all subjects. There were no 
significant differences between the hemispheres for the onset latency of the RFs or for the peak latency and magnitude of the MFs and MEFs (RF onset time, $\mathrm{p}=0.796$; MF latency, $\mathrm{p}=$ 0.342; MEF latency, $\mathrm{p}=0.919 ;$ MF amplitude, $\mathrm{p}=0.108$; MEF amplitude, $\mathrm{p}=0.956$ ). The peak latency of SEFs ranged from $46.0 \mathrm{~ms}$ to $87.2 \mathrm{~ms}$ (mean, $68.2 \mathrm{~ms}$ ) by tongue stimulation.

\subsection{Source localizations}

The isofield contour maps are shown at the peak latency of the MFs, MEFs, and SEFs in Fig. 3A. The orientations of the ECDs were directed anteriorly for the MFs (Fig. 3A [1]) but posteriorly for the MEFs (Fig. 3A [2]) and SEFs (Fig. 3A [3]). The ECDs producing the MFs were located in the anterior bank of the central sulcus, suggesting the location of the tongue M1 (Fig. 3B). In contrast, the ECDs for the MEFs and SEFs were located in the posterior bank of the central sulcus, suggesting the location of the tongue S1.

These locations were similar across the group of subjects. The mean coordinates of each component were $x=-43.9 \mathrm{~mm}, y=23.4 \mathrm{~mm}$, and $z=87.8 \mathrm{~mm}$ for the MFs; $x=$ $-51.3 \mathrm{~mm}, y=15.8 \mathrm{~mm}$, and $z=74.5 \mathrm{~mm}$ for the MEFs; and $x=-55.9 \mathrm{~mm}, y=17.9$ $\mathrm{mm}$, and $z=75.1 \mathrm{~mm}$ for the SEFs. The analysis of variance revealed a significant main effect for the ECD location in each axis ( $x$-axis, $\mathrm{p}<0.001 ; y$-axis, $\mathrm{p}=0.006$; $z$-axis, $\mathrm{p}<$ 0.001). The statistical analysis indicated that the ECDs for the MFs were located significantly medial (mean, $12.0 \mathrm{~mm}$; $\mathrm{p}=$ 0.006, Bonferroni corrected for multiple comparisons), anterior (mean, $5.4 \mathrm{~mm} ; \mathrm{p}=0.030$ ), and superior (mean, $12.7 \mathrm{~mm} ; \mathrm{p}=$ 0.001) to those for the SEFs. The ECDs for the MEFs were located significantly medial (mean, $4.6 \mathrm{~mm} ; \mathrm{p}=0.005$, Bonferroni corrected for multiple comparisons) to those for 
the SEFs, but not significantly posterior (mean, $2.1 \mathrm{~mm}$; $\mathrm{p}=0.905$ ) or inferior (mean, $0.7 \mathrm{~mm} ; \mathrm{p}=1.00$ ) (Fig. 4). Moreover, the ECDs for the MFs were located significantly medial (mean, $7.4 \mathrm{~mm} ; \mathrm{p}=$ 0.041, Bonferroni corrected for multiple comparisons) and superior (mean, $13.3 \mathrm{~mm}$; $\mathrm{p}=0.021$ ) to those for the MEFs, but not significantly anterior (mean, $7.6 \mathrm{~mm} ; \mathrm{p}=0.063$ ). The relative ECD locations of the MFs and MEFs in comparison with the SEF locations in each subject are summarized on axial and coronal planes in Fig. 4.

\section{Discussion}

The present study demonstrated the components of MRCFs over both hemispheres occurring before and after self-paced tongue movements. Importantly, the MF component, which followed the pre-movement RF component, originated from the bilateral M1, suggesting that the bilateral M1 is involved in the preparation and execution of tongue movements. Moreover, the post-movement MEF component originated from the bilateral S1, suggesting that the MEF may reflect proprioceptive feedback from the tongue during tongue movement.

Previous MRCF studies accompanying voluntary unilateral finger movements generally agree that the slow RF component precedes the movement and appears mainly in the contralateral hemisphere (Deecke et al., 1982; Cheyne and Weinberg, 1989; Kristeva et al., 1991; Nagamine et al., 1996; Babiloni et al., 2001). In contrast, here, the RF appeared in both hemispheres during tongue movements, without hemispheric dominance in the onset latency, peak latency, or peak magnitude. Since both sides of the tongue were moving symmetrically during the forward tongue movement task, and since each side of the tongue is innervated by bilateral corticobulbar fibers through the 
hypoglossal nucleus, it is reasonable that we observed cortical activation in both hemispheres to self-paced tongue protrusion. The lack of hemispheric dominance in the pre-movement cortical activation for tongue protrusion was consistent with our previous study using corticomuscular coherence (CMC) analyses in the beta frequency band. In that study, we found that the functional connection was contralateral dominant for each tongue side, but the contralateral connection between the cortex and tongue showed no hemispheric lateralization for either tongue side during isometric tongue protrusion (Maezawa et al., 2014c).

It has been shown that the pre-movement slow components detected by EEG/MEG (Bereitschaftspotential [BP] in EEG; RF in MEG) are associated with the preparation and execution of voluntary finger movement (Shibasaki and Hallett, 2006). Transcranial magnetic stimulation studies in humans demonstrated that at about 80 to 120 ms prior to the onset of voluntary finger movements, the excitability of pyramidal-tract neurons starts to elevate (Chen et al., 1998; Chen and Hallett, 1999; Leocani et al., 2000). This suggests that the RF onset occurs prior to the excitation of pyramidal-tract neurons. Given the fact that pyramidal-tract neurons are directly involved in the execution of finger movements, the RF early phase may be related to both the execution of movements and to the programming or preparation of movements. Thus, the detection of the RF component over both hemispheres during tongue movement suggests that the bilateral tongue M1 may be involved in programming and/or preparing voluntary tongue movements as well as controlling them.

The post-movement MEF component was also detected over both hemispheres after movement onset in all subjects. As reported by Gerloff et al. (1998), steady-state movement-related magnetic fields (ssMRMFs) occur during self-paced, fast, repetitive 
finger movements at $2 \mathrm{~Hz}$. After EMG onset, two main ssMRMF components were observed: an MF component, which peaked at $37 \mathrm{~ms}$ and had an anterior current orientation, and a post-movement field (post-MF) component, which peaked at $102 \mathrm{~ms}$ and had a posterior current orientation. Compared to the MF component from M1, the post-MF component originating from S1 had much larger amplitude. These findings indicated that the MF component represented a corticospinal volley from M1, whereas the post-MF component was related to reafferent feedback processing from the muscle spindles to S1 (Gerloff et al., 1998). Regarding the similarities in the peak latency and current orientation between the post-MF and MEF of the MRCF during finger movements, the MEF may reflect a similar component to the post-MF (Gerloff et al., 1998). Moreover, Ikeda et al. (1995) reported movement-related potentials from subdural electrodes over the lower perirolandic area of the side with the lesion during tongue protrusions in patients with epilepsy. The post-movement component of the reafferent potential, which had a positive polarity, was located in the tongue region of $\mathrm{S} 1$, in contrast to the pre-movement component of CMC, which originated from M1. Thus, given that the MEF may reflect the same component as the reafferent potential observed in the subdural recordings, the observed tongue MEFs over both hemispheres in our study may indicate that the proprioceptive feedback information is processed from the muscle spindles of the tongue to bilateral S1 during tongue protrusion. In fact, our recent study using $\mathrm{CMC}$ analyses suggested that the $\mathrm{CMC}$ at the low frequency band may be driven by proprioceptive afferents from the tongue muscles to bilateral S1 (Maezawa et al., 2016c). Especially, since the muscle spindles of the tongue are highly developed in humans compared to those in other primates, such sensory processing over both hemispheres may play an important role in the fine-tuning of tongue movements. 
It is currently unclear why the ECDs for the MEFs were located significantly medial to those for the SEFs in the present study. It is possible that the location differences may be because of the different generator mechanisms between MEFs and SEFs; specifically, MEFs may reflect the cortical activation related to proprioceptive sensation and SEFs may reflect the activation related to cutaneous sensation. It is reasonable that the potential generators of the MEFs are located in area 3a, since area 3a in $\mathrm{S} 1$ receives the initial proprioceptive response from muscle spindles. However, given the fact that the current orientation in area 3a is mainly radial, it was difficult to detect the response in area 3a using MEG. Thus, we conclude that the main region of MEFs may be area $3 b$ in S1, which receives initial activation from area 3a. Moreover, it should be acknowledged that the SEFs induced by tongue stimulation in the present study were not the initial component of area 3b. Previous MEG studies reported that the initial component of tongue SEFs was hard to detect owing to its small amplitude; this is in contrast with the middle-latency component of tongue SEFs, which is easy to detect given its large amplitude. Nonetheless, the precise mechanisms of the middle-latency component of tongue SEFs are not well understood. Further studies are needed to reveal the precise generator mechanism of MEFs and to compare the location of MEFs to that of the initial component of tongue SEFs.

In conclusion, the present results indicate that the MF and MEF components are generated from M1 and S1, respectively. We conclude that the MF likely reflects a generator mechanism in bilateral M1 for the preparation and control of voluntary tongue movement. In contrast, the MEF may represent proprioceptive afferent processing from the tongue muscle to bilateral S1. Here, the cortical processes related to the efferent and afferent information for voluntary tongue protrusion in humans were successfully 
analyzed using whole-head MEG with a high spatiotemporal resolution.

\section{Conflicts of interest}

None of the authors have potential conflicts of interest to be disclosed.

\section{Acknowledgements}

This work was supported by Grants-in-Aid for Scientific Research (C)25462883 (MF) and Grants-in-Aid for Young Scientists (B)25862071 (HM) from the Japan Society for the Promotion of Science. 


\section{References}

Babiloni, F., Carducci, F., Cincotti, F., Del Gratta, C., Pizzella, V., Romani, G.L., Rossini, P.M., Tecchio, F., Babiloni, C., 2001. Linear inverse source estimate of combined EEG and MEG data related to voluntary movements. Hum. Brain. Mapp. 14, 197-209.

Chen, R., Yaseen, Z., Cohen, L.G., Hallett, M., 1998. Time course of corticospinal excitability in reaction time and self-paced movements. Ann. Neurol. 44, 317325.

Chen, R., Hallett, M., 1999. The time course of changes in motor cortex excitability associated with voluntary movement. Can. J. Neurol. Sci. 26, 163-169.

Cheyne, D., Weinberg, H., 1989. Neuromagnetic fields accompanying unilateral finger movements: pre-movement and movement-evoked fields. Exp. Brain. Res. 78, $604-612$.

Deecke, L., Scheid, P., Kornhuber, H.H., 1969. Distribution of readiness potential, pre-motion positivity, and motor potential of the human cerebral cortex preceding voluntary finger movements. Exp. Brain. Res. 7, 158-168.

Deecke, L., Weinberg, H., Brickett, P., 1982. Magnetic fields of the human brain accompanying voluntary movement: Bereitschaftsmagnetfeld. Exp. Brain. Res. 48, 144-148.

Gerloff, C., Uenishi, N., Nagamine, T., Kunieda, T., Hallett, M., Shibasaki, H., 1998. Cortical activation during fast repetitive finger movements in humans: steady-state movement-related magnetic fields and their cortical generators. Electroencephalogr. Clin. Neurophysiol. 109, 444-453. 
Hämäläinen, R., Hari, R., Ilmoniemi, R., Knuutila, J., Lounasmaa, O.V., 1993. Magnetoencephalography—-theory, instrumentation, and applications to noninvasive studies of the working human brain. Rev. Mod. Phys. 65, 413-497. Ikeda, A., Lüders, H.O., Burgess, R.C., Sakamoto, A., Klem, G.H., Morris, HH. $3^{\text {rd }}$, Shibasaki, H., 1995. Generator locations of movement-related potentials with tongue protrusions and vocalizations: subdural recording in human. Electroencephalogr. Clin. Neurophysiol. 96, 310-328.

Kristeva, R., Cheyne, D., Deecke, L., 1991. Neuromagnetic fields accompanying unilateral and bilateral voluntary movements: topography and analysis of cortical sources. Electroenceph. Clin. Neurophysiol. 81, 284-298.

Leocani, L., Cohen, L.G., Wassermann, E.M., Ikoma, K., Hallett, M., 2000. Human corticospinal excitability evaluated with transcranial magnetic stimulation during different reaction time paradigms. Brain 123, 1161-1173.

Maezawa, H., Yoshida, K., Nagamine, T., Matsubayashi, J., Enatsu, R., Bessho, K., Fukuyama, H., 2008. Somatosensory evoked magnetic fields following electric tongue stimulation using pin electrodes. Neurosci. Res. 62, 131-139.

Maezawa, H., Yoshida, K., Matsuhashi, M., Yokoyama, Y., Mima, T., Bessho, K., Fujita, S., Nagamine, T., Fukuyama, H., 2011. Evaluation of tongue sensory disturbance by somatosensory evoked magnetic fields following tongue stimulation. Neurosci. Res. 71, 244-250.

Maezawa, H., Hirai, Y., Shiraishi, H., Funahashi, M., 2014a. Somatosensory evoked magnetic fields following tongue and hard palate stimulation on the preferred chewing side. J. Neurol. Sci. 347, 288-294. 
Maezawa, H., Matsuhashi, M., Yoshida, K., Mima, T., Nagamine, T., Fukuyama, H., 2014b. Evaluation of lip sensory disturbance using somatosensory evoked magnetic fields. Clin. Neurophysiol. 125, 363-369.

Maezawa, H., Mima, T., Yazawa, S., Matsuhashi, M., Shiraishi, H., Hirai, Y., Funahashi, M., 2014c. Contralateral dominance of corticomuscular coherence for both sides of the tongue during human tongue protrusion: an MEG study. Neuroimage 101, 245-255.

Maezawa, H., Onishi, K., Yagyu, K., Shiraishi, H., Hirai, Y., Funahashi, M., 2016a. Modulation of stimulus-induced $20-\mathrm{Hz}$ activity for the tongue and hard palate during tongue movement in humans. Clin. Neurophysiol. 127, 698-705.

Maezawa, H., Tojyo, I., Yoshida, K., Fujita, S., 2016b. Recovery of impaired somatosensory evoked fields after improvement of tongue sensory deficits with neurosurgical reconstruction. J. Oral. Maxillofac. Surg. 74,1473-1482.

Maezawa, H., Mima, T., Yazawa, S., Matsuhashi, M., Shiraishi, H., Hirai, Y., Funahashi, M., 2016c. Cortico-muscular synchronization by proprioceptive afferents from the tongue muscles during isometric tongue protrusion. Neuroimage 128, 284 292.

Nagamine, T., Kajola, M., Salmelin, R., Shibasaki, H., Hari, R., 1996. Movement-related slow cortical magnetic fields and changes of spontaneous MEG- and EEG-brain rhythms. Electroencephalogr. Clin. Neurophysiol. 99, 274-286.

Nakasato, N., Itoh, H., Hatanaka, K., Nakahara, H., Kanno, A., Yoshimoto, T., 2001. Movement-related magnetic fields to tongue protrusion. Neuroimage 14, 924935. 
Oishi, M., Kameyama, S., Fukuda, M., Tsuchiya, K., Kondo, T., 2004. Cortical activation in area 3b related to finger movement: an MEG study. Neuroreport 15, $57-62$.

Shibasaki, H., Barrett, G., Halliday, E., Halliday, A.M., 1980. Components of the movement-related cortical potential and their scalp topography. Electroencephalogr. Clin. Neurophysiol. 49, 213-226.

Shibasaki, H., Hallett, M., 2006. What is the Bereitschaftspotential? Clin. Neurophysiol. 117, 2341-2356.

Shibukawa, Y., Shintani, M., Kumai, T., Suzuki, T., Nakamura, Y., 2004. Cortical neuromagnetic fields preceding voluntary jaw movements. J. Dent. Res. 83, $572-577$.

Taulu, S., Simola, J., 2006. Spatiotemporal signal space separation method for rejecting nearby interference in MEG measurements. Phys. Med. Biol. 51, 1759-1768.

Taulu, S., Hari, R., 2009. Removal of magnetoencephalographic artefacts with temporal signal-space separation: demonstration with single-trial auditory-evoked responses. Hum. Brain. Mapp. 30, 1524-1534.

Tesche, C.D., Uusitalo, M.A., Ilmoniemi, R.J., Huotilainen, M., Kajola, M., Salonen, O., 1995. Signal-space projections of MEG data characterize both distributed and well-localized neuronal sources. Electroencephalogr. Clin. Neurophysiol. 95, 189-200.

Uusitalo, M.A., Ilmoniemi, R.J., 1997. Signal-space projection method for separating MEG or EEG into components. Med. Biol. Eng. Comput. 35, 135-140.

Vaughan, H.G. Jr., Costa, L.D., Ritter, W., 1968. Topography of the human motor potential. Electroencephalogr. Clin. Neurophysiol. 25, 1-10. 
Table 1. Onset latency, peak latency, and peak magnitude of the movement-related cortical fields in each hemisphere.

\begin{tabular}{|c|c|c|c|c|c|c|c|c|c|c|}
\hline \multirow[b]{3}{*}{$S$} & \multirow{2}{*}{\multicolumn{2}{|c|}{$\begin{array}{c}\text { Onset latency } \\
\text { of RF (s) }\end{array}$}} & \multicolumn{4}{|c|}{ Peak latency (ms) } & \multicolumn{4}{|c|}{ Peak magnitude (fT/cm) } \\
\hline & & & \multicolumn{2}{|c|}{ MF } & \multicolumn{2}{|c|}{ MEF } & \multicolumn{2}{|c|}{ MF } & \multicolumn{2}{|c|}{ MEF } \\
\hline & $\mathrm{Lt}$ & Rt & $\mathrm{Lt}$ & Rt & $\mathrm{Lt}$ & Rt & $\mathrm{Lt}$ & $\mathrm{Rt}$ & $\mathrm{Lt}$ & $\mathrm{Rt}$ \\
\hline 1 & -1.13 & -1.67 & -29 & -25 & 161 & 209 & 38.4 & 42.1 & 68.9 & 72.1 \\
\hline 2 & -0.20 & -0.25 & 28 & 3 & 119 & 130 & 28.4 & 37.8 & 29.1 & 73.2 \\
\hline 3 & -0.48 & -0.19 & 48 & 52 & 142 & 165 & 101.8 & 100.8 & 66.6 & 47.5 \\
\hline 4 & -0.63 & -0.45 & 45 & -29 & 153 & 159 & 43.8 & 31.2 & 54.2 & 45.2 \\
\hline 5 & -1.63 & -0.34 & 37 & 41 & 214 & 175 & 69.4 & 34.3 & 67.3 & 59.0 \\
\hline 6 & -1.52 & -1.34 & -1 & 26 & 161 & 161 & 57.4 & 26.4 & 43.3 & 40.9 \\
\hline 7 & -0.53 & -1.39 & 37 & 6 & 240 & 200 & 58.9 & 39.7 & 55.2 & 43.5 \\
\hline$M$ & -0.874 & -0.804 & 23.5 & 10.4 & 169.9 & 171.4 & 56.87 & 44.61 & 54.94 & 54.49 \\
\hline SD & 0.554 & 0.633 & 28.4 & 31.2 & 42.3 & 26.6 & 24.16 & 25.34 & 14.65 & 13.67 \\
\hline
\end{tabular}

RF, Readiness field; MF, Motor field; MEF, Motor evoked field; S, Subject number; Lt, Left hemisphere; Rt, Right hemisphere; M, Mean; SD, Standard deviation. 


\section{Figure legends}

Figure 1. The whole-head magnetic waveforms of movement-related cortical fields (MRCFs) associated with self-paced tongue protrusion in Subject 5. The vertical lines show the onset of tongue electromyography (EMG). Each trace starts $3 \mathrm{~s}$ before and ends $1 \mathrm{~s}$ after EMG onset. The MRCFs were detected over the sensorimotor areas on both sides. The dotted circles indicate the waveforms with the maximum amplitude for each MRCF component (motor fields [A, C] and motor evoked fields [B, D]) over both hemispheres. The details are described in Fig. 2.

Figure 2. Enlarged magnetic waveforms from representative channels over the left and right hemispheres (dashed circle traces A-D in Fig. 1) and the rectified and averaged electromyography (EMG) signal associated with self-paced tongue protrusion in Subject 5. The vertical lines show the onset of tongue EMG. Each trace starts $3 \mathrm{~s}$ before and ends $1 \mathrm{~s}$ after EMG onset. A slow pre-movement component of the readiness fields (RFs) started prior to movement onset bilaterally (A, left hemisphere; C, right hemisphere) and culminated in the motor fields (MFs) just after movement onset. Movement evoked fields (MEFs) appeared bilaterally after movement onset (B, left hemisphere; D, right hemisphere). MEG, Magnetoencephalography.

Figure 3. A. Isocontour maps over the left hemisphere for the motor fields (MFs) and the movement evoked fields (MEFs) associated with tongue protrusion, as well as the somatosensory evoked fields (SEFs) following right tongue stimulation in Subject 5. The contour map was obtained from the time points showing waveform peaks for each component. Dashed and solid lines indicate the outgoing and incoming magnetic fluxes, 
respectively. Gray arrows show the location and direction of the equivalent current dipoles (ECDs) projected on the skull surface. Arrowheads indicate the negative pole of the ECDs. Note that the directions of the ECDs were anterior for the MF [1] but posterior for the MEF [2] and SEF [3]. The contour steps are $20 \mathrm{fT}$ (MFs), $20 \mathrm{fT}$ (MEFs), and $10 \mathrm{fT}$ (SEFs). B. ECD locations over the left hemisphere for the MF, MEF, and SEF in Subject 5. The ECDs of the MF were located in the anterior part of the central sulcus, suggesting the primary motor cortex. The ECDs of the MEF and SEF were located in the posterior part of the central sulcus, suggesting the primary somatosensory cortex.

Figure 4. Top row: Equivalent current dipole (ECD) locations of the motor fields (MFs) and movement evoked fields (MEFs) relative to the locations of somatosensory evoked fields (SEFs) in all subjects on axial (left panel) and coronal planes (right panel). The ECDs of the MFs are located significantly medial, anterior, and superior to the SEFs. The ECDs of the MEFs are located significantly medial but not anterior or superior to the SEFs. ECD locations of the MFs and MEFs are connected with bars in each subject. Bottom row: ECD locations for SEFs of Subject 5 are superimposed on axial (left panel) and coronal (right panel) magnetic resonance images. The SEF location was used as the coordinate origin in each subject to compare it with the MF and MEF locations. All locations are in mm. 


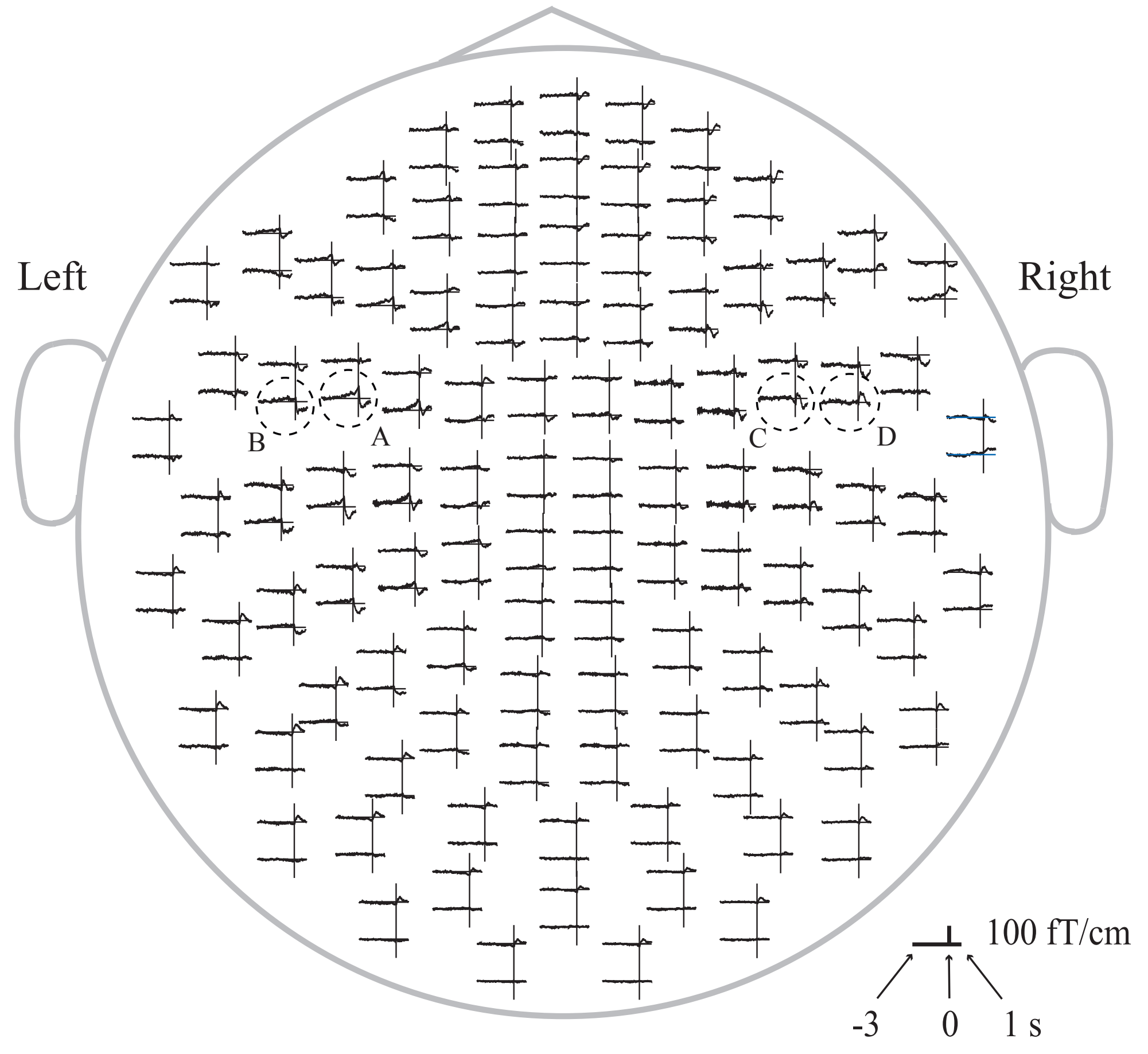


MEG

A

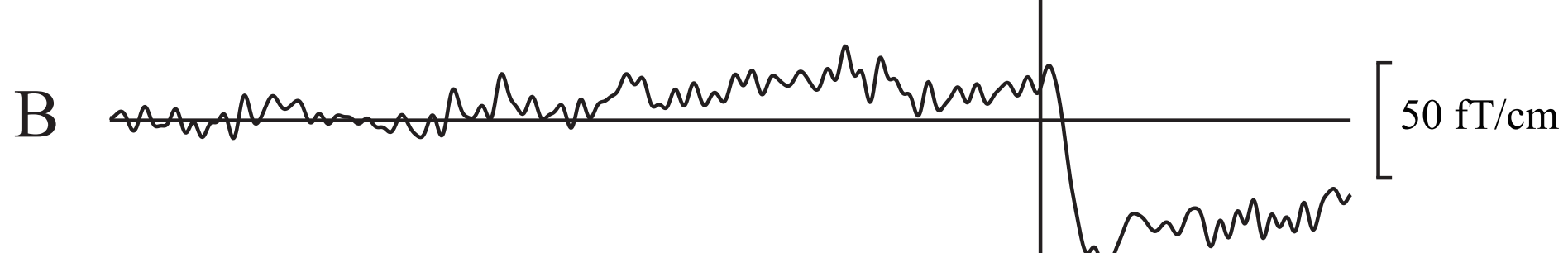

Right

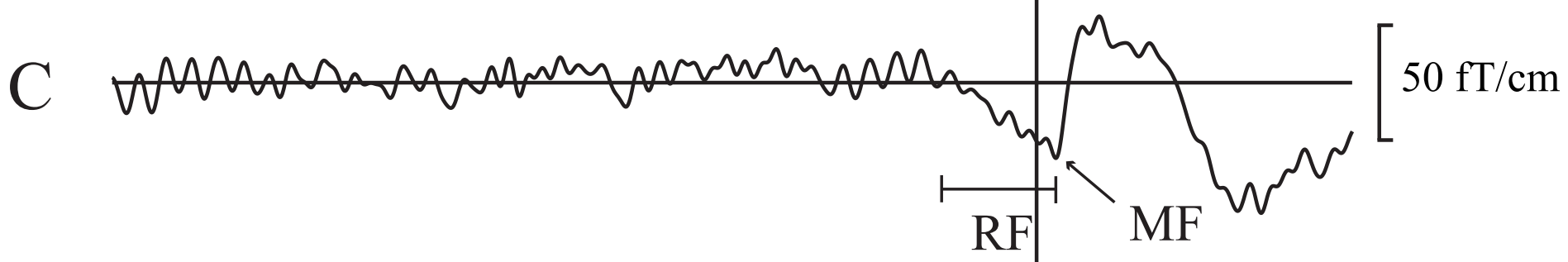

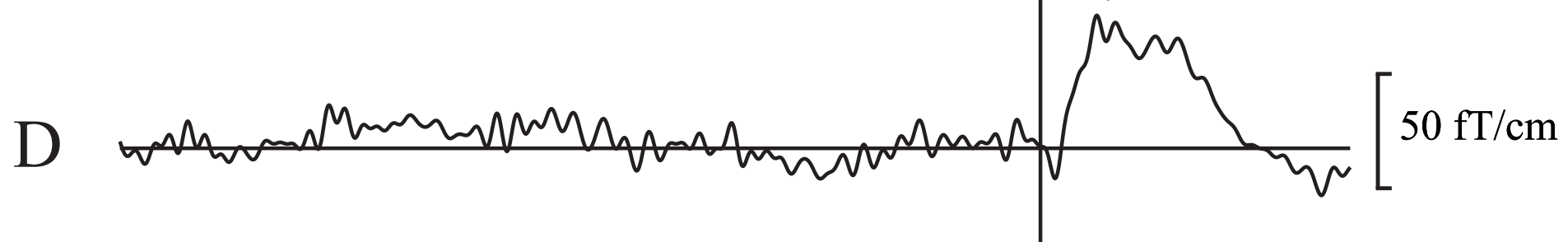

EMG
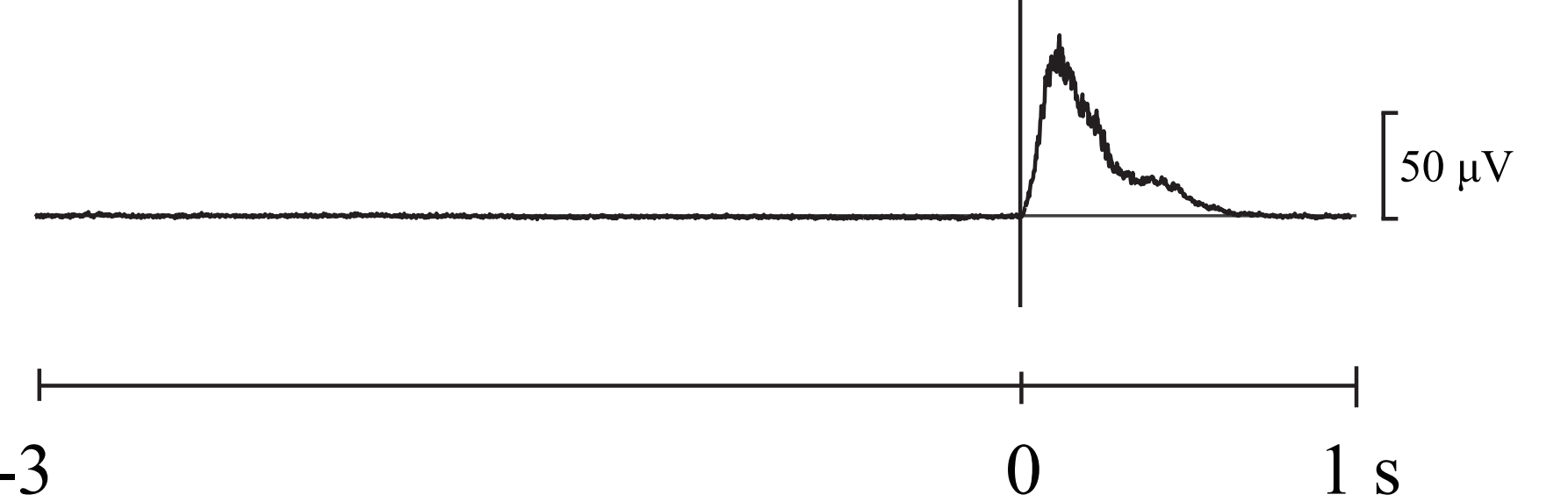
A. Isocontour maps

[1] MFs

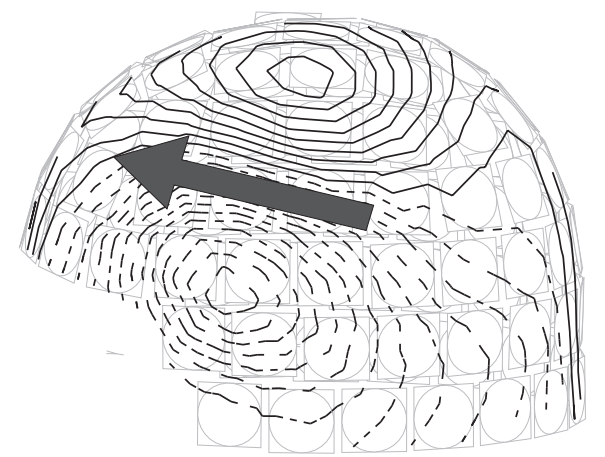

[2] MEFs

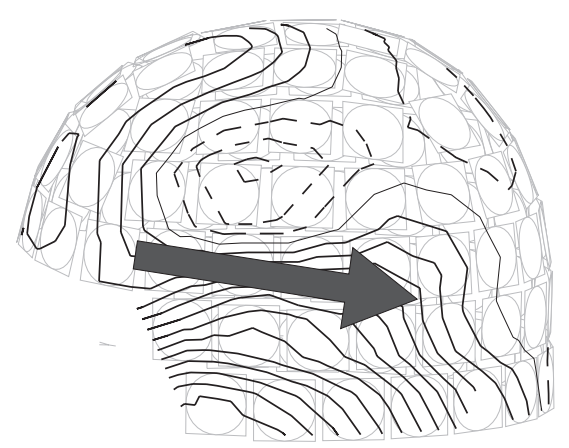

[3] SEFs

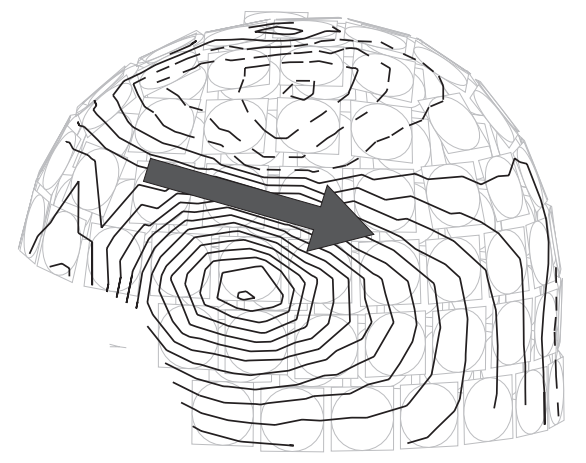

B. Dipole locations and orientations of the MRCF and SEF

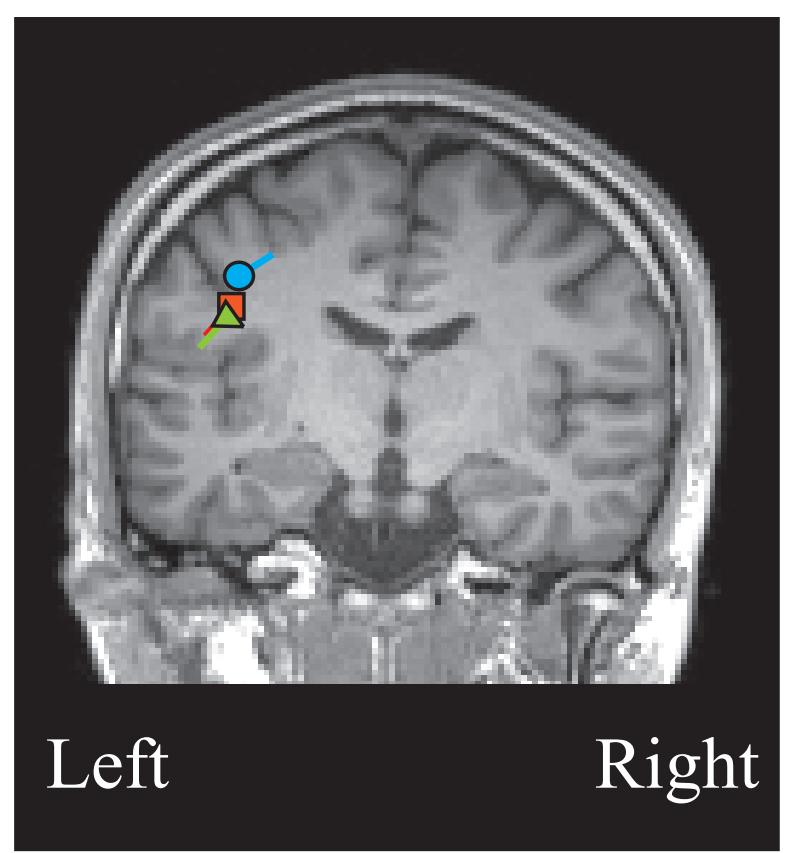

○ $\mathrm{MF}$

$\square \mathrm{MEF}$

$\triangle \mathrm{SEF}$

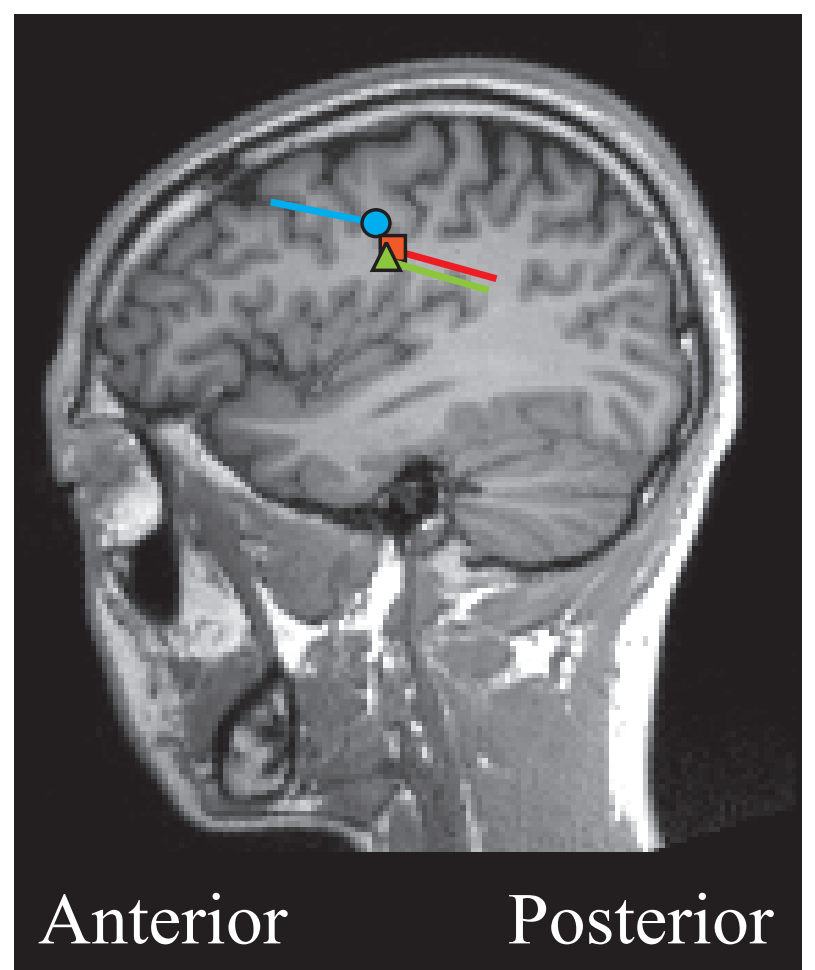

Anterior

\section{Posterior}



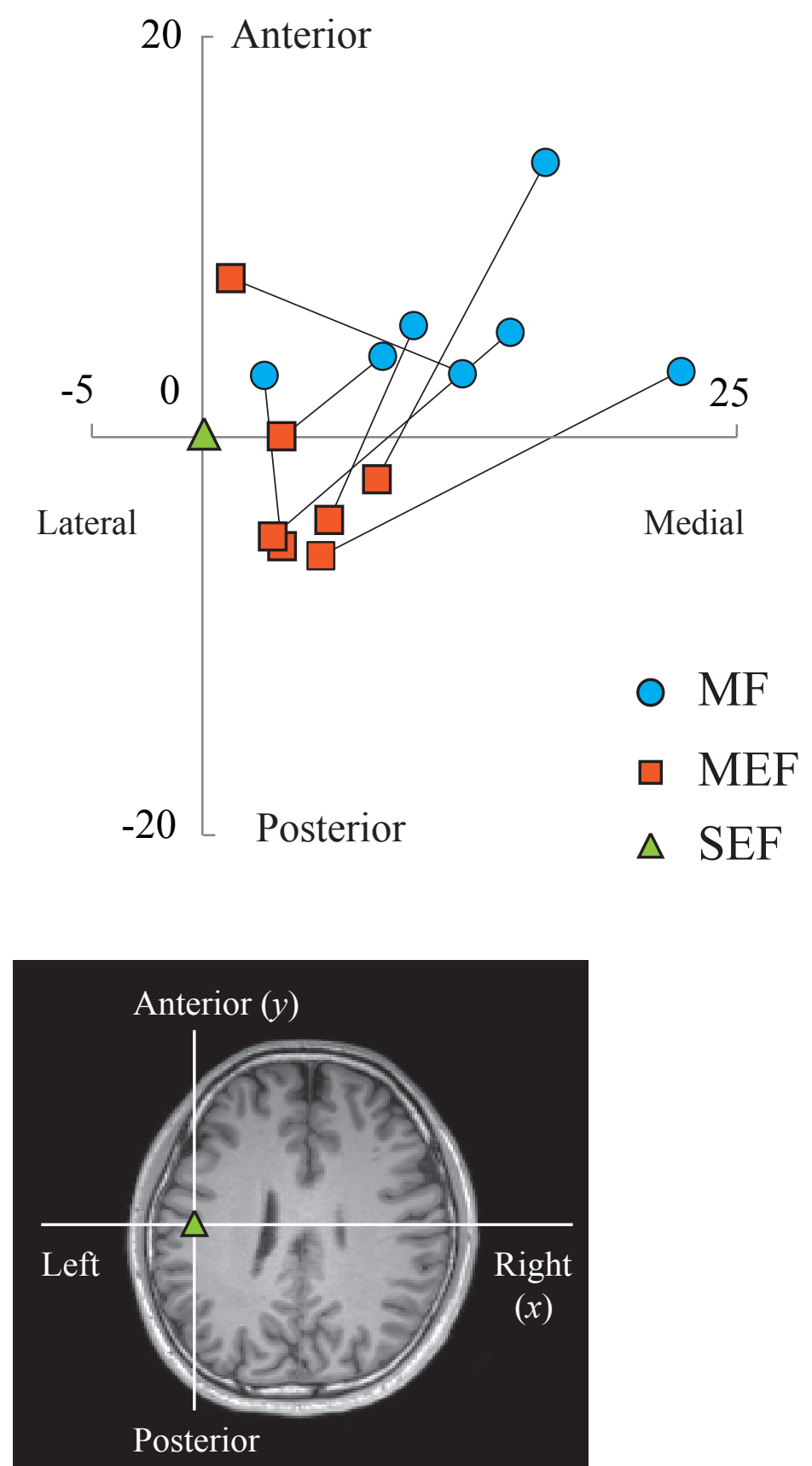
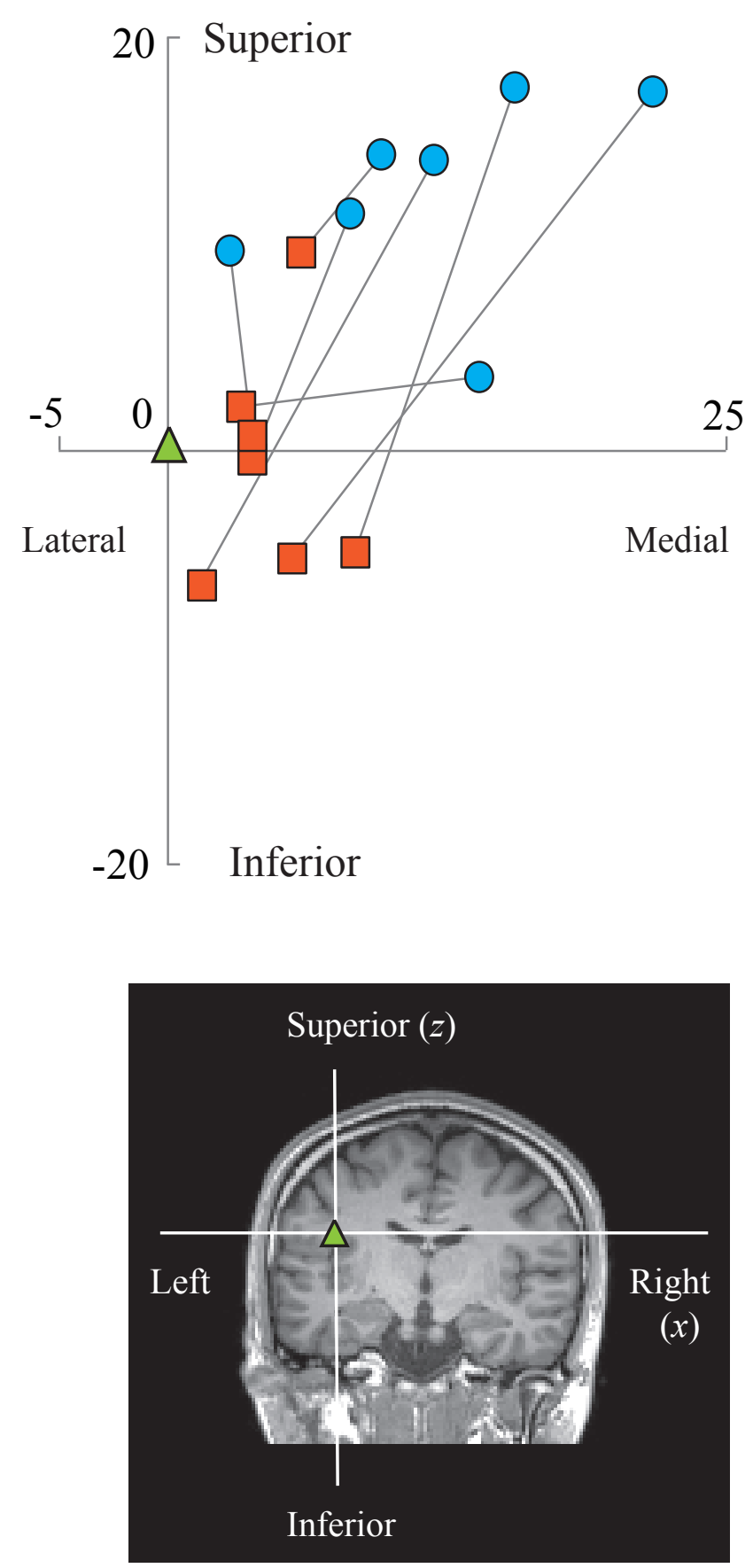